\title{
Image Identification Based on Shape and Color Descriptors and Its Application to Ornamental Leaf
}

\author{
Kohei Arai, Indra Nugraha Abdullah, Hiroshi Okumura \\ Graduate School of Science and Engineering, Saga University, Saga, Japan
}

\begin{abstract}
Human has a duty to preserve the nature, preserving the plant is one of the examples. This research has an emphasis on ornamental plant that has functionality not only as ornament but also as medicine. Although in Indonesia, in general this plant is cultivated in front of the house; only few people know about its medicinal function. Considering this easiness to obtain and its medicinal function, this plant has to be an initial treatment or option towards full chemical-based medicines. This research proposes a system which able to identify properly ornamental plant from its leaf utilizing its shape or color features. Shape descriptor represented by Dyadic Wavelet Transformation and Zernike Complex Moment, and HSV-based color histogram as color descriptor. This research provides benefit of these three methods to solve various test aspects. It was obtained $81.77 \%$ of overall averagetesting performance.
\end{abstract}

Index Terms —Identification, Dyadic wavelet, Zernike mo ments, HSV, SVM, leaf, overlapping

\section{INTRODUCTION}

Oxygen is an essential part for all living things in the world. Plant plays an important role to produce oxygen and supply it for their sustainable life. The cycle between human and the plant is the interesting one. Carbon dioxide as the output of human respiratory is needed by plant for photosynthesis activity. Then, this activity is resulting oxygen which vital for human. According to this cycle, human supposed to preserve the plant to maintain availability of oxygen.

Based on International Union for Conservation of Nature and Natural Resources, the number of identified plant species in the world which consist of Mosses (M), Ferns and Allies (FA), Gy mnosperms, Flowering Plants (FP), Red and Green Algae (RGA) is about 307.674 species [1].

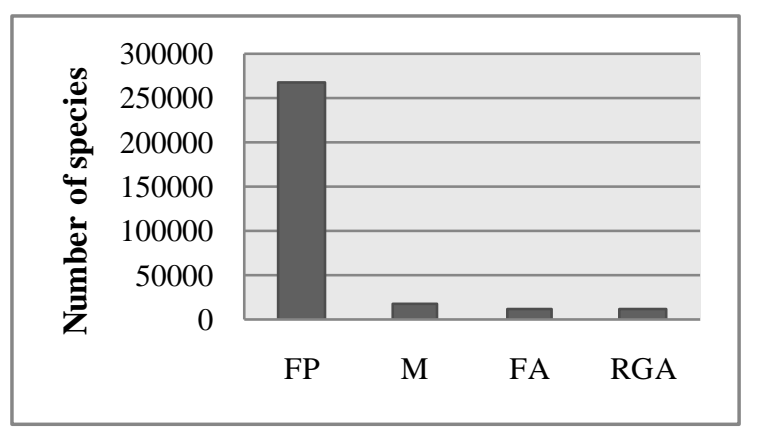

Figure.1 Column charts number of identified plant species

On the other side, the approximate number of unidentified species is 86.429 species. It consists of Flowering Plants with 83.400 species, Ferns and Allies with 3.000 species, Mosses with 29 species [2]. Considering the highest number possessed by Flowering Plants, identification of the plants, which include also ornamental plant, has become a challenge for us.

As a recognition step of the unidentified species, this research has an emphasis on ornamental leaf which has medicinal function. Although mainly in Indonesia this plant is cultivated in front of the house; only few people know about its medicinal function. If this easiness and its medicinal function are taken into consideration, this plant has to be an initial treatment or option towards full chemical-based medicines.

Identification of leaf image is possibly done through identification of some leaf features, i.e. shape, venation, and color. Nevertheless, most of the researchers were using shape feature to identify the leaf. Shape is a substantial part to describe image content. Naturally, we lost one dimension of in formation while projecting of a real world three-dimensional object onto the twodimensional projection plane. This reason makes the shape features are often only represent projected object not real world object. Proper methods as the solution of real world object problems are needed.

The representation of shape can be separated into contour-based and region-based. Both representations can be further separated into structural and global [3]. Dyadic wavelet transformation and Zernike complex moments are representing global method of contourbased approach, and global method of region-based 
approach, respectively. We are expecting nice representation of shape using a combination from contour-based and region-based.

Formerly, many researchers had conducted image processing using Dyadic wavelet transformation. As in [4] and [5], they were applying this method for image denoising purpose. The results for both researchers were satisfying. In [7], they were adopting Zernike complex moments to classify benign and malignant masses on Mammogram images. The moments were divided into two groups. First group was low-order of the moments, and second was high-order of the moments. It was concluded that low-order magnitude of Zernike complex mo ment was preferred in comparis on to high-order.

In leaf identification area, Park, Hwang, and Nam [11] had developed image retrieval system for leaf images collection. Utilization of leaf venation became their primary point to identify the leaf. Then, followed by the work that proposed by Wang et.al. [13], they classified leaf image with complicated background. Segmentation process of the leaf utilizing automatic marker for Watershed method, and Classification process was done by combining $7 \mathrm{Hu}$ moments and 16 Zernike moments. This work inspires us to be further developed in segmentation and moment area. The color feature is also important to identify the leaf. In real life, frequently, the identification process is affected by aspect, i.e. lighting changed from the sun. We hope utilization of HSV can guarantee us to identify the leaf properly.

In this paper, we describe the ornamental leaf in the section 2, followed by the proposed method for image identification based on shape and color descriptors, flowchart of this research in the section 4, information of classification in the section 5, and the experiment and results as the final section.

\section{ORNAMENTAL LEAF AS MEDICINA L LEAF}

Ornamental leaf in this research is not general ornamental leaf. Besides the function of ornamental leaf as an ornament in an open space, also can be used as herbal medicine, which cure basic disease.

Image data set of ornamental leaf in this research was obtained from direct acquisition using a digital camera. This data set is taken based on tropical ornamental plant that usually cultivated in front of the house in Indonesia. This data set contains 8 classes with 15 images for each class. The classes are Bay (syzygium polyanthum), Cananga (canagium odoratum, lamk), Mangkokan (nothopanax scutellarium merr.), Jas mine (jasminum sambac [soland]), Cocor bebek (kalanchoe pinnuta), Vinca (catharanthus roseus), Kestuba (euphorbia pulcherrima, willd), Gardenia (gardenia augusta, merr). In order to avoid expensiveness of computation, size of the image is $256 \times 256$ pixels. The sample images of each class are presented as follows:

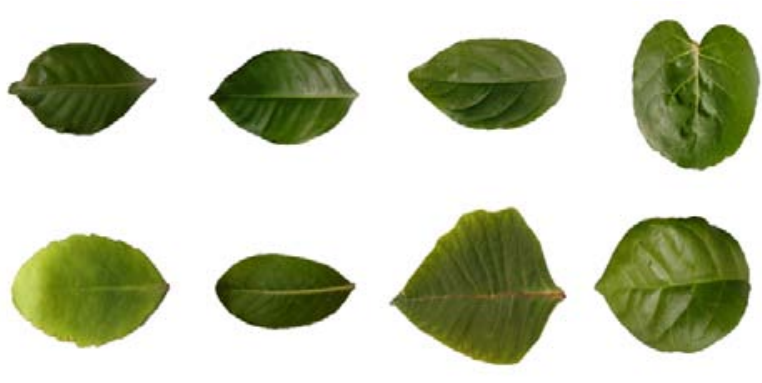

Figure.2 Sample Image of each class inside data set

The table.1 shows specific medicinal function of corresponding ornamental leaf. Most of them, ways to apply are the leaf is boiled together with water, and then apply as drinks, the other example is for skin cure, the leaf s mears directly to the skin [14].

\section{TABLE.1 MEDICINAL FUNCTION OF ORNAMENTAL} LEAF

\begin{tabular}{|l|l|}
\hline Name & Medicinal function \\
\hline Bay & Diarrhea, scabies and itching \\
\hline Cananga & Asthma \\
\hline Mangkokan & Mastitis, skin injury, hair loss \\
\hline Jasmine & Fever, head ache, sore ey es \\
\hline Cocor Bebek & Ulcer, diarrhea, gastritis \\
\hline Vinca & Diabetes, fever, burn \\
\hline Kestuba & Bruise, irregular menstrual \\
\hline Gardenia & Sprue, fever, constipation \\
\hline
\end{tabular}

\section{IDENTIFICATION BASED ON SHAPE A ND COLOR DESCRIPTORS}

\section{A Dyadic wavelet transform}

The downsampling wavelet, which samples the scale and translation parameters, is often failing when deal with some assignments such as edge detection, features extraction, and image denoising [5]. Different with the downsampling wavelet, the dyadic wavelet samples only the scale parameter of a continuous wavelet transform, and does not samples the translation factor. In one side, it creates highly redundant signal representation, but the other side, since it has the shift-invariance ability, this method is a convincing candidate as a shape descriptor method.

Let $L^{2}(R)$ be the space of square integrable functions on real line $R$, and define the Fourier transform of the function $\psi \in L^{2}(R)$ by 


$$
\widehat{\psi}(\omega)=\int_{-\infty}^{\infty} \psi(t) e^{-i \omega t} d t
$$

If there is exist $A>0$ and $B$ such that

$$
A \leq \sum_{-\infty}^{\infty}\left|\widehat{\psi}\left(2^{j} \omega\right)\right|^{2} \leq B
$$

then $\psi(t)$ is called dyadic wavelet function. Dyadic wavelet transform of $f(t)$ is defined using this $\psi(t)$ by

$$
W f\left(u, 2^{j}\right)=\int_{-\infty}^{\infty} f(t) \frac{1}{\sqrt{2^{j}}} \psi\left(\frac{t-u}{2^{j}}\right),
$$

from (2), $\widehat{\psi}(0)=0$ must be satisfied, i.e., $\int_{-\infty}^{\infty} \psi(t) d t=$ 0 . In order to design the dyadic wavelet function, we need a scaling function $\phi(t)$ satisfying a two-scale relation

$$
\phi(t)=\sum_{k} h[k] \sqrt{2} \phi(2 t-k) .
$$

The scaling function $\phi(t)$ is usually normalized as $\int_{-\infty}^{\infty} \phi(t) d t=1$.

By (4), the Fourier transform of the scaling function resulting

$$
\hat{\phi}(\omega)=\frac{1}{\sqrt{2}} \hat{h}\left(\frac{\omega}{2}\right) \hat{\phi}\left(\frac{\omega}{2}\right),
$$

where $\hat{h}$ denotes a discrete Fourier transform

$$
\widehat{h}(\omega)=\sum_{k} h[k] e^{-i \omega t}
$$

since $\hat{\phi}(0)=1$, we can apply (5) and (6) to obtain $\widehat{h}(0)=\sqrt{2}$. Using the scaling function and the wavelet filter $g[k]$, we define a dyadic function by $\psi(t)=$ $\sum_{k} g[k] \sqrt{2} \phi(2 t-k)$.

The Fourier transform of $\psi(t)$,

$$
\hat{\psi}(\omega)=\frac{1}{\sqrt{2}} \hat{g}\left(\frac{\omega}{2}\right) \hat{\phi}\left(\frac{\omega}{2}\right)
$$

will be needed later.

Let us denote the discrete Fourier transform of the filters $h[k], h[k], \tilde{h}[k]$, and $\tilde{g}[k]$, by $h(\omega), h(\omega), \hat{h}(\omega)$ and $\widehat{\tilde{g}}(\omega)$ respectively. We suppose that these Fourier transforms satisfy the below condition

$$
\hat{h}(\omega) \hat{h}^{*}(\omega)+\hat{g}(\omega) \hat{g}(\omega)=2, \omega \in[-\pi, \pi],
$$

where * denotes complex conjugation. This condition called a reconstruction condition.

Under condition (8), we have

$$
a_{j+1}[n]=\sum_{k} h[k] a_{j}\left[n+2^{j} k\right] ; j=0,1, \ldots,
$$

$$
d_{j+1}[n]=\sum_{k} g[k] a_{j}\left[n+2^{j} k\right] ; j=0,1, \ldots
$$

here $a_{0}[n]$ is given by $a_{0}[n]=\int_{-\infty}^{\infty} f(t) \phi(t-n) d t$. The (9) and (10) are dyadic decomposition formula for one-dimensional signals.

Shape descriptor from this method is represented by variance, standard deviation and centroid. The details are start from approximation, horizontal, vertical, and diagonal details will have these representation numbers. The following image shows the details:

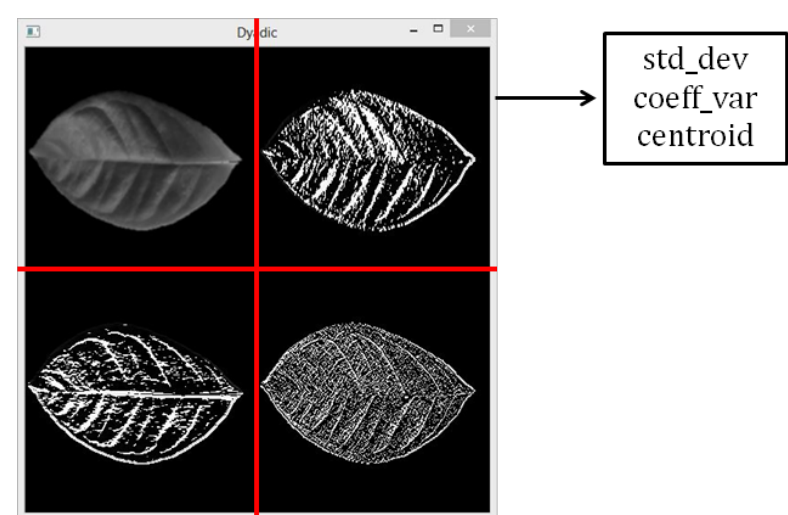

Figure.3 Representation of dy adic wavelet transformed image

\section{B Zernike complex moments}

The following steps are steps to calculate Zernike mo ments:

1. Computation of radial polyno mials

2. Computation of Zernike basis functions

3. Computation of Zernike moments through projecting the image onto its basis functions

First step is computation of radial polynomial and represented by the following equation:

$$
\mathrm{R}_{\mathrm{n}}^{\mathrm{m}}(\rho)=\sum_{\mathrm{k}=0}^{(\mathrm{n}-\mathrm{m}) / 2} \frac{(-1)^{\mathrm{k}}(\mathrm{n}-\mathrm{k}) !}{\mathrm{k} !\left(\frac{\mathrm{n}+\mathrm{m}}{2}-\mathrm{k}\right) !\left(\frac{\mathrm{n}-\mathrm{m}}{2}-\mathrm{k}\right) !} \rho^{\mathrm{n}-2 \mathrm{k}}
$$

$n$ and $m$ are representing the order and the repetition of azimuthal angle, respectively. The length of vector from original point to point $(x, y)$ denoted by $\rho$.

From (11), complex two-dimensional Zernike basis functions are constructed by:

$$
V_{n, m}(\rho, \theta)=R_{n}^{m}(\rho)^{\mathrm{j} m \theta}, \quad|\rho| \leq 1
$$

The following orthogonality condition is satisfied by the complex Zern ike polynomials: 


$$
\begin{aligned}
& \int_{0}^{2 \pi} \int_{0}^{1} V_{n, m}^{*}(\rho, \theta) V_{p, q}(\rho, \theta) \rho d \rho d \theta \\
& =\left\{\begin{array}{cl}
\frac{\pi}{n+1} & ; n=p, m=q \\
0 & ; \text { otherwise }
\end{array}\right.
\end{aligned}
$$

Sign * denotes complex conjugation. This orthogonality defines no redundancy inter-mo ments with different order and repetitions.

Here is the definition of the complex Zernike mo ments

$$
Z_{n, m}=\frac{n+1}{\pi} \int_{0}^{2 \pi} \int_{0}^{1} f(\rho, \theta) V_{n, m}^{*}(\rho, \theta) \rho d \rho d \theta
$$

Image function, $f(a, b)$, for digital images can be replaced by summation operation.

The discrete Zernike moments for image with size $\mathrm{N} x$ $\mathrm{N}$ is defined as

$$
\begin{aligned}
Z_{n, m}= & \frac{n+1}{\lambda_{N}} \sum_{a=0}^{N-1} \sum_{b=0}^{N-1} f(a, b) V_{n, m}^{*}(a, b) \\
= & \frac{n+1}{\lambda_{N}} \sum_{a=0}^{N-1} \sum_{b=0}^{N-1} f(a, b) R_{n}^{m}\left(\rho_{a b}\right) e^{-j m \theta_{a b}}
\end{aligned}
$$

Where $\lambda_{\mathrm{N}}$ is normalization factor and $0 \leq \rho_{\mathrm{ab}} \leq 1$. Transformed distance $\rho_{\mathrm{ab}}$ and the phase $\theta_{\mathrm{ab}}$ at the pixel position $(\mathrm{a}, \mathrm{b})$ are showed with following equations:

$$
\begin{aligned}
& \rho_{\mathrm{ab}}=\frac{\sqrt{(2 \mathrm{a}-\mathrm{N}+1)^{2}+(2 \mathrm{~b}-\mathrm{N}+1)}}{N} \\
& \theta_{\mathrm{ab}}=\tan ^{-1}\left(\frac{N-1-2 a}{2 a-N+1}\right)
\end{aligned}
$$

The magnitude of Zernike moments from original image with rotated image will be equal.

This rotation-invariant ability became a reason to propose this method as another shape descriptors. This research only used low-order of Zernike complex moments because of computation inexpensive and not sensitive to the noise $[7,15]$. The following table shows the proposed low-order Zern ike complex moments:

TABLE.2 LOW-ORDER ZERNIKE COMPLEX MOMENTS

\begin{tabular}{|c|c|c|}
\hline Order $n$ & Iteration $\mathrm{m}$ & $\begin{array}{c}\text { Number of } \\
\text { moments }\end{array}$ \\
\hline 3 & 1,3 & 2 \\
\hline 4 & $0,2,4$ & 3 \\
\hline 5 & $1,3,5$ & 3 \\
\hline
\end{tabular}

\begin{tabular}{|c|c|c|}
\hline 6 & $0,2,4,6$ & 4 \\
\hline 7 & $1,3,5,7$ & 4 \\
\hline 8 & $0,2,4,6,8$ & 5 \\
\hline 9 & $1,3,5,7,9$ & 5 \\
\hline 10 & $0,2,4,6,8,10$ & 6 \\
\hline \multicolumn{3}{|c|}{ Total moments 32} \\
\hline
\end{tabular}

\section{HSV-based color histogram}

The general use of color space RGB only defines color using a combination of primary color. Different with RGB, HSV color space defines color similar to how the human eye tends to perceive color. HSV color space is hexacone with center vertical axis is intensity [19]. Hue represents color type, and it consists of red at angle 0 , green at $2 \pi / 3$, blue $4 \pi / 3$, and red again at $2 \pi$. Saturation is depth of color and measured as radial distance from the central axis to the outer surface.

In this research, we are not proposing to use global approach histogram where one whole image is measured by one histogram. We propose to use local approach, which one whole image is separated into 4 parts and measure the histograms. As an illustration, refer to Fig.4.

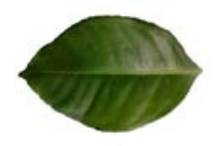

(a)

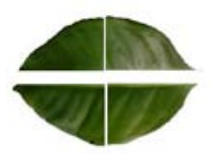

(b)
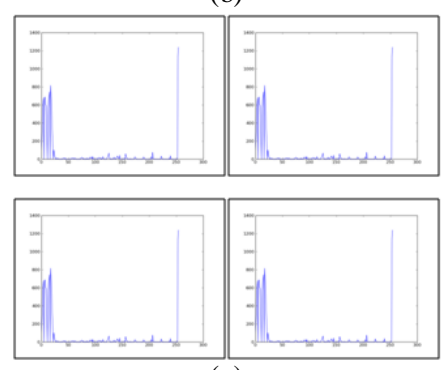

(c)

Figure.4 Local approach color histogram. (a) original image, (b) sliced images, (c) histogram of each slice

\section{FLOW CHART}

The following image is the flowchart of this research. 


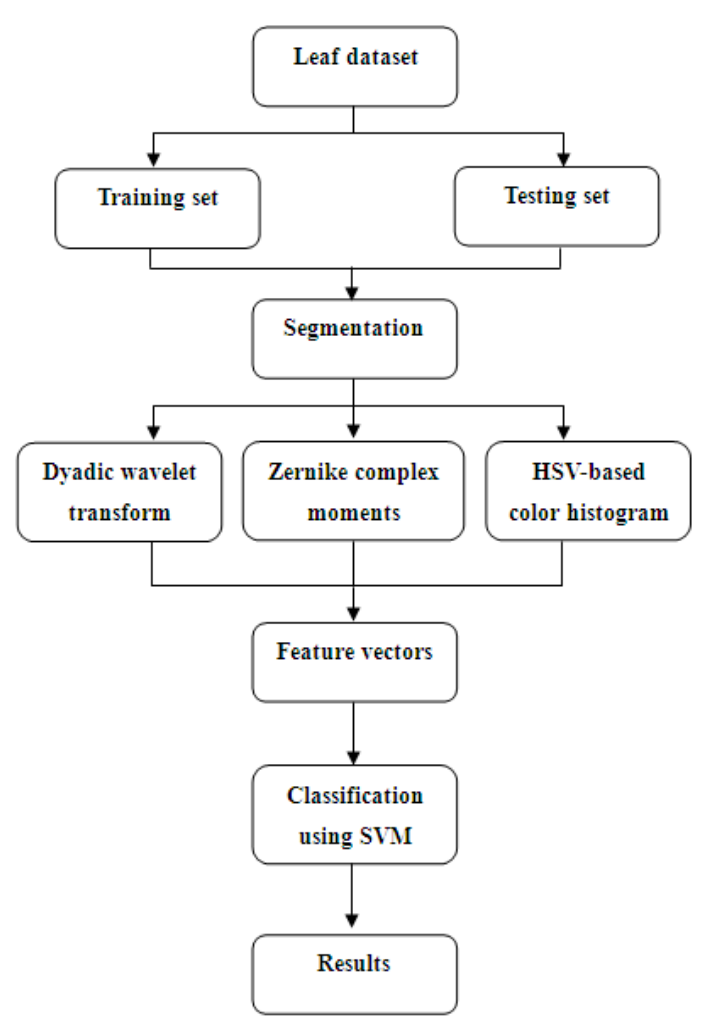

Figure.5 Flowchart of this research

Leaf data set is separated into training and testing sets, respectively. Image is segmented to remove unnecessary background object and obtain the foremost leaf for overlapping image. Next step is feature extraction using three methods. First, dyadic wavelet transformation, second is Zernike complex moment, both are as shape descriptors, and last is HSV-based color histogram. The data classification that extracted from these methods is classified utilizing SVM classifier. The classification process is involving two corresponding methods, classification towards the training set, and classification towards the testing set, respectively.

\section{CLASSIFICATION}

\section{A SVM classifier}

SVM is a powerful tool for data classification. The indicators are the easiness to apply and impose Structural Risk Minimization (SRM). SRM made the SVM has strong ability in generalization of data. Its function is to minimize an upper bound on the expected risk. In principle, SVM learns to obtain optimal boundary with maximu m marg in that able to separate set of objects with different class of membership.

In order to achieve the maximum margin classifier, we have two options. Hard margin and soft margin are the options that totally depend on linearity of the data. Hard margin SVM is applicable to a linearly separable data set. However, often the data is not linearly separable. Soft margin SVM emerged as its solution $[16,17]$. The optimization problem for the soft margin SVM presented as below:

$$
\begin{gathered}
\min _{w, \xi, b} \frac{1}{2}\|w\|^{2}+C \sum_{i=1}^{n} \xi_{i} \\
\text { subject to: } y_{i}\left(w^{T} x_{i}+b\right)-1+\xi_{i} \geq 0, \quad \xi_{i} \\
\geq 0 .
\end{gathered}
$$

where $w, C, \xi, b$ are the weight vectors, the penalty of misclassification or margin errors, the margin error, the bias, respectively.

In (18) can lead us to an efficient kernel method approach. A kernel method is an algorithm that depends on the data only through kernel function, which computes a dot product in some possibly high dimensional data. Using the function $\phi$ training vector, the input space $x$ is mapped into higher dimensional space. $K\left(x_{i}, x_{j}\right)=\phi\left(x_{i}\right)^{T} \phi\left(x_{j}\right)$ is called kernel function. The degree of the polynomial kernel can control the flexibility of resulting classifier [17]. It will be appropriate with this research when we classify 8 classes of leaf. Polynomial kernel is shown in equation (19).

$$
K\left(x_{i}, x_{j}\right)=\left(\gamma x_{i}{ }^{T} x_{j}+r\right)^{d}, \gamma>0 .
$$

Where $\gamma, r, d$ are kernel parameters, and $i, j$ denote $i^{\text {th }}, j^{\text {th }}$ vector in data set.

In this research, we propose to use Sequential Minimal Optimization (SMO). SMO act as efficient solver of the optimization problem in the training of support vector machines. SMO also solves the problems analytically by way of breaks the problems into a series of smallest possible problems.

\section{B Various test aspects}

In relation to measure the performance comprehensively, we are involving various test aspects. These aspects covered rotation, scaling, translation, lighting and perspective aspects. The following image is showed for visualization purpose:

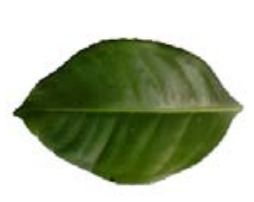

(a)

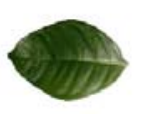

(c)

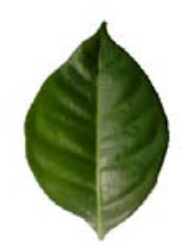

(b)

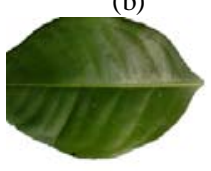

(d) 


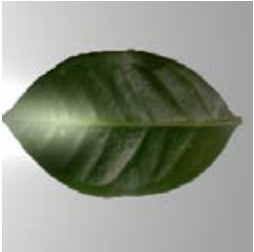

(e)

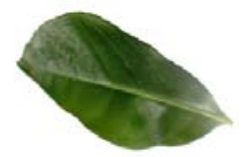

(f)
Figure.6 Various test aspects. (a) original image, (b) rotated, (c) scaled, (d) translated, (e) lighting chan ged, (f) perspective chan ged.

\section{EXPERIMENT AND RESULT}

\section{A Segmentation results}

Here are steps of segmentation for this data set:

1. Create structuring element with the anchor point is the middle of the image

2. Do a erosion operation to obtain 2 separated marker for next step

3. Choose the biggest eroded shape as a target

4. Set the markers; the biggest shape as foreground and smaller shape as background.

5. Do a watershed segmentation using above markers

6. Single foremost leaf is obtained

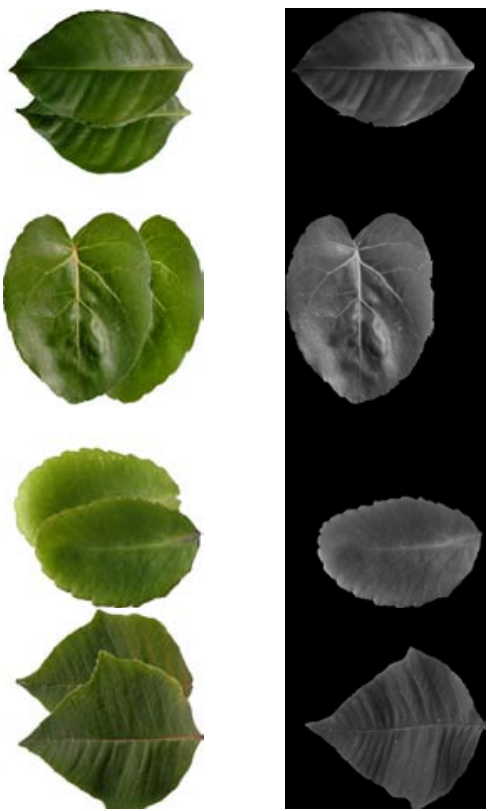

Figure.7 Segmentation results using Watershed method. Left images are original overlapping leaves and right images is segmented single leaf
There are limitations of segmentation using this method. In this research, the position of anchor point is set manually which made this method cannot solve full arbitrary overlapped regions. The other is the number of overlapping leaves must be equal with two. However, through this method a real life problem such as segmentation besides of above points can be properly solved.

\section{B Classification performances}

1) Decomposition level selection of dyadic wavelet transform

Selection of best decomposition level for this leaf image data set is based on three test data set. Original data set consists of leaf images without any changes, rotation data set consists of leaf images with rotation changes, and translation data set consists of leaf images with translation changes.

TABLE.3 CLASSIFICATION FOR LEVEL SELECTION PURPOSE (IN PERCENT)

\begin{tabular}{|l|c|c|c|}
\hline \multicolumn{1}{|c|}{ Data set } & level 1 & level 2 & level 3 \\
\hline Original & 94.79 & 95.83 & 94.79 \\
\hline Rotation & 92.59 & 85.19 & 88.89 \\
\hline Translation & 100.00 & 100.00 & 100.00 \\
\hline Average & 95.79 & 93.67 & 94.56 \\
\hline
\end{tabular}

Level denotes decomposition level, and from the experiments were obtained that decomposition level 1 was the suitable level. This level will be used for further classification measurement. Besides the level, the table. 3 has shown that Dyadic wavelet transformation is translation-invariant.

\section{2) Overall performances}

Color distribution inside the data set is only limited to green. In this research, we only use a small number of bins. Hue and saturation bins were 4 bins and 6 bins. Therefore, one histogram has 24 bins and one whole image has 96 bins as a representation of color descriptor.

TABLE.4 CONTRIBUTION FROM COMBINATION OF EVERY DESCRIPTOR (IN PERCENT)

\begin{tabular}{|c|c|c|c|c|}
\hline Method & Dyadic + Zernike & Dyadic + Histo & Zernike + Histo & $\begin{array}{c}\text { Dyadic + Zernike + } \\
\text { Histo }\end{array}$ \\
\hline Trainingset + test set & 81.25 & 62.5 & 81.25 & 93.75 \\
\hline
\end{tabular}


TABLE.5 CLASSIFICATION PERFORMANCES TOWARD TEST ASPECTS (IN PERCENT)

\begin{tabular}{|l|c|c|c|c|}
\hline Method & $\begin{array}{c}\text { Rotation, Translation, } \\
\text { Scaling (1) }\end{array}$ & $\begin{array}{c}\text { (1)+Lighting } \\
\text { changed (2) }\end{array}$ & $\begin{array}{c}\text { (1)+Perspective } \\
\text { changed (3) }\end{array}$ & $\begin{array}{c}\text { Combination } \\
(1+2+3)\end{array}$ \\
$\begin{array}{l}\text { Training } \\
\text { set (a) }\end{array}$ & 100 & 100 & 100 & 100 \\
\hline $\begin{array}{l}\text { (a)+Test } \\
\text { set }\end{array}$ & 93.75 & 75 & 87.5 & 70.83 \\
\hline
\end{tabular}

The number of training and testing sets for the experiments were 13 images and 2 images, respectively. Table.4 is presented as comparison for contribution from every combination of descriptors. Dyadic, Zernike, and Histo denote dyadic wavelet transformation, Zernike complex moments, and HSV-based color histogram, respectively. The best performance was gained from the combination of shape and color descriptors.

As shown in table.5, this system was able to identify the data of the entire training sets and created a proper model showed by number $100 \%$ of all training set field. The cooperation from shape descriptors that represented by Dyadic wavelet transformation and Zernike complex moments was resulting excellent results in the rotation, translation and scaling data set indicating by $93.75 \%$ of supplied test-set result. The changes in perspective view of two-dimensional projection plan were affected only to differentiation of the shape itself. Moreover, SVM classifier also gave a positive contribution separated the data through their polynomial kernel function.

Different with the other aspects, in the lighting aspect, although we found that color descriptor plays an important role as a representation of a leaf image, dominant part of the shape is being distorted because of this change. This issue could not properly be solved by this system. However, the overall results of previously mentioned test aspects were fine.

\section{CONCLUSION}

It is found that combination between shape descriptors from Dyadic wavelet transformation and Zernike complex moments that emphasis on scaling, translation and rotation-invariant were resulting fine results. Because coupled with HSV-based color descriptor, this system could solve lighting invariance problem, though the result was not perfect. Future works from this current research are development of lifting scheme of Dyadic wavelet transformation, for the sake of easiness of calculation and memory efficient reasons, implements fast Zernike moments computation in connection with the recursive calculation of radial polynomial, and find another proper method to overcome arbitrary lighting change problems.

\section{REFERENCES}

[1] IUCN 2012. Numbers of threatened species by major groups of organisms (1996-2012). $<$ http://www.iucnredlist.org/documents/summaryst atistics/2012_2_RL_Stats_Table_1.pdf >. Downloaded on 21 June 2013.

[2] Chapman A. D. Numbers of Living Species in Australia and the World. Australian Government, Department of the Environment, Water, Heritage, and the Arts. Canberra, Australia, 2009.

[3] Zhang D., Lu G. Review of shape representation and description techniques. Pattern Recognition, 2004, vol.37, 1-19.

[4] Abdukirim T., Nijima K., Takano S. Lifting dyadic wavelets for denoising. Proceedings of the Third International Workshop on Spectral Methods and Multirate Signal Processing (SMMSP), 2003, 147154.

[5] Minamoto T., Tsuruta K., Fujii S. Edge-preserving image denoising method based on dyadic lifting schemes. IPSJ Transactions on Computer Vision and Applications, 2010, vol. 2, 48-58.

[6] Mallat S. A wavelet tour of signal processing. Academic Press. 1998.

[7] Tahmasbi A., Saki F., Shokouhi S. B. Classification of benign and malignant masses based on zernike moments. Computers in Biology and Medicine, 2011, vol. 41, 726-735.

[8] S.K. Hwang, W.Y. Kim, A novel approach to the fast computation of Zernike moments, Pattern Recognition, 2006, vol.39, 2065-2076.

[9] Ch. Y. Wee1, R. Paramesran, F. Takeda. Fast computation of zernike moments for rice sorting system. Proceedings of the IEEE, International Conference on Image Processing (ICIP), 2007, pp. VI-165-VI-168.

[10] B. Fu, J. Liu, X. Fan, Y. Quan. A hybrid algorithm of fast and accurate computing zernike moments. Proceedings of the IEEE, International Conference on Fuzzy Systems and Knowledge Discovery (FSKD), 2007, pp. 268-272.

[11] Park Jinkyu, Hwang Eenjun, Nam Yunyoung. Utilizing venation features for efficient leaf image retrieval. The Journal of System and Software, 2008, Vol.81, 71-82.

[12] Du Jin-Xiang, Wang Xiao-Feng, Zhang Guo-Jun. Leaf Shape based plant species recognition. 
Applied Mathematics and Computation, 2007, vol. 185, 883-893.

[13] Wang Xiao-Feng, et.al. Classification of plant images with complicated background. Applied Mathematics and Computation, 2008, vol.205, 916926.

[14] Hartati Sri. Tanaman hias berkhasiat obat. IPB Press. 2011. (In Indonesian)

[15] C.H. Theh, R.T. Chin, On image analysis by the methods of mo ments, IEEE Transactions on Pattern Analysis and Machine Intelligence, 1988, vol. 4(10), 496-513.

[16] Ben-Hur Asa, Weston Jason. A user's guide to support vector machine. Data Mining Techniques for the Life Science, 223-239, Humana Press, 2010.

[17] Hsu Chih-Wei, Chang Chih-Chuang, Lin Chih-Jen. A practical guide to support vector classification. Department of Computer Science National Taiwan University, Taiwan, 2010.

[18] Starck Jean-Luc, Murtagh Fionn, Fadili Jalal M. Sparse image and signal processing. Cambridge University Press. 2010.

[19] G.Stockman and L.Shapiro. Computer Vision. Prentice Hall, 2001.

Kohei Arai received BS, MS and PhD degrees in 1972, 1974 and 1982, respectively. He was with The Institute for Industrial Science and Technology of the University of Tokyo from April 1974 to December 1978 and also was with National Space Development Agency of Japan from January, 1979 to March, 1990. During from 1985 to 1987, he was with Canada Centre for Remote Sensing as a Post Doctoral Fellow of National Science and Engineering Research Council of Canada. He moved to Saga University as a Professor in Department of Information Science on April 1990. He was a counselor for the Aeronautics and Space related to the Technology Committee of the Ministry of Science and Technology from 1998 to 2000. He was a counselor of Saga University for 2002 and 2003. He also was an executive counselor for the Remote Sensing Society of Japan for 2003 to 2005. He is an Adjunct Professor of University of Arizona, USA since 1998. He also is Vice Chairman of the Commission A of ICSU/COSPAR since 2008.

Indra Nugraha Abdullah was born at Bogor, Indonesia. Finished his bachelor degree in Bogor Agricultural University and graduated from Saga University for master degree in the field of Information Science on March 2011. He is currently pursuing to get Ph.D. Degree from the same university with specialization in image processing area. Leaf identification becomes his interest in his latest degree.

Hiroshi Okumura was born at Kyoto, Japan in 1964. He received B.E.S.E. and M.E.S.E. degree from Hosei University in 1988 and 1990, respectively, and Ph.D degree on environmental engineering from Chiba
University in 1993. He became a research associate at Remote Sensing and Image Research Center, Chiba University first in 1993. Next, he became a research associateand a lecturer at the Department of Electrical Engineering, Nagaoka University of Technology in 1995 and 2000, respectively. He is now an associate professor at the Department of Information Science, Saga University. His research interests are in image and speech processing and remote sensing. 